\title{
T Cells in the Lesion of Experimental Autoimmune Encephalomyelitis Enrichment for Reactivities to Myelin Basic Protein and to Heat Shock Proteins
}

Felix Mor and Irun R. Cohen

Department of Cell Biology, The Weizmann Institute of Science, Rehovot, 76100, Israel

\section{Abstract}

To characterize the cellular immune response in an autoimmune lesion, we investigated the accumulation of specific $T$ cells in the central nervous system in actively induced experimental autoimmune encephalomyelitis (EAE) in Lewis rats, using a limiting dilution analysis (LDA) assay for $T$ cells that proliferate in response to antigens. Lymphocytes isolated from the spinal cord infiltrate were compared with cells from the popliteal lymph nodes with respect to frequency of cells responding to basic protein (BP), mycobacterium tuberculosis (MT), the 65-kD heat shock protein (hsp65), allogeneic brown norway spleen cells, and concanavalin A. Additionally, we compared the BP frequency in acute EAE of cells from the spinal cord, peripheral blood, spleen and lymph nodes, and the spinal cord and lymph node after recovery from EAE. We found that acute EAE was associated with marked enrichment of BP-reactive $T$ cells in the spinal cord relative to their frequency in the lymphoid organs and peripheral blood. The infiltrate was also enriched for $\mathrm{T}$ cells responding to $\mathrm{hsp65}$; alloreactive $\mathrm{T}$ cells, in contrast, were not enriched. The frequency of BP reactive $T$ cells in the spinal cord was highest at the peak of paralysis; however, BP-reactive $T$ cells could still be detected at moderate frequencies after clinical recovery. We established BP- and Mycobacteria-reactive $T$ cell lines from the spinal infiltrates that were $\mathrm{CD4}^{+}$and TcR $\alpha \beta^{+}$. Most of the BP lines were found to react to the major encephalitogenic epitope of guinea pig BP for rats (amino acids 71-90); these lines were found to mediate EAE in naive recipients. $T$ cell lines recognizing other epitopes of BP were not encephalitogenic. All of the lines responsive to Mycobacteria recognized hsp65 or hsp70. These results indicating that the immune infiltrate in active EAE is enriched with cells responding to the autoantigen and to hsp65 were confirmed in EAE adoptively transferred by anti-BP T cell clone. (J. Clin. Invest. 1992.90:2447-2455.) Key words: autoimmune disease $\bullet$ immunopathology $\bullet$ limiting dilution analysis $\bullet$ neuroantigens $\bullet T$ cell lines

\section{Introduction}

Experimental autoimmune encephalomyelitis (EAE) ${ }^{1}$ is a prototype autoimmune disease mediated by $\mathrm{T}$ cells. The dis-

1. Abbreviations used in this paper: $\mathrm{BP}$, basic protein; CFA, complete Freund's adjuvant; EAE, experimental autoimmune encephalomyelitis; hsp, heat shock protein; LDA, limiting dilution analysis; MT, Mycobacterium tuberculosis; TCGF, T cell growth factor.

Address reprint requests to Prof. I. R. Cohen, Department of Cell Biology, The Weizmann Institute of Science, P.O. Box 26, Rehovot 76100, Israel.

Received for publication 26 December 1991 and in revised form 7 May 1992

J. Clin. Invest.

(c) The American Society for Clinical Investigation, Inc.

0021-9738/92/12/2447/09 \$2.00

Volume 90, December 1992, 2447-2455 ease is induced in genetically susceptible strains of mice and rats by the inoculation of myelin basic protein (BP) or proteolipid protein in complete Freund's adjuvant (CFA) (1). This immunization leads to the generation of effector $T$ cells responding to BP that migrate to the central nervous system (CNS) and cause the disease (2). Immunization of rats to BP in incomplete Freund's adjuvant induces anti-BP T cells (3), but without the dead Mycobacteria in the adjuvant the immunized rats do not develop clinical EAE. Thus the Mycobacteria in the CFA facilitate the active induction of EAE.

In the last 30 years several groups of investigators have attempted to study the nature and the specificity of the autoimmune infiltrate in EAE (4-8). Early studies based on adoptive transfer of radiolabeled primed lymph node cells led to the conclusion that the majority of the lymphocytes in the brain are not specific for BP (4) and that the blood-brain barrier is permeable to lymphocytes without respect to their antigen specificity (5). Yet other studies pointed to a preferential accumulation of specific $T$ cells in the lesion $(2,6)$. In the one study that examined directly the frequency of BP-responsive cells in the spinal cord, a similar frequency was found in the primed popliteal lymph nodes (7). Recently, Hickey et al. (8) analyzed the kinetics of cell migration into the CNS after intravenous inoculation of encephalitogenic and control cells (8). These authors concluded that activated $T$ cells irrespective of antigen specificity penetrate the CNS within hours of injection, however, only BP-specific cells are retained in the nervous tissue $72 \mathrm{~h}$ after inoculation (8).

We conducted the present study to examine the question of the frequency of BP-responsive T cells in the EAE lesion. We also investigated whether the EAE lesion might be enriched in $\mathrm{T}$ cells responsive to heat shock protein (hsp) antigens. Hsp65 is interesting because it is a dominant antigen in the Mycobacteria present in the CFA and because $T$ cells responsive to hsp65 have been found to function in autoimmune arthritis (9) and in autoimmune diabetes $(10,11)$. We also studied alloreactive $T$ cells because such $T$ cells are not known to cause autoimmune disease and they are detectable in high frequency without the need for priming.

The results of this study indicate that the CNS infiltrate is highly enriched for encephalitogenic anti-BP T cells but also accumulates $T$ cells reactive to hsp65. Alloreactive $T$ cells, in contrast, were present in the lesions at the same frequency as in the lymph nodes. The frequency of BP-reactive $T$ cells was highest at the peak of paralysis; but these $T$ cells also could be found in the lesion at lower frequencies long after clinical recovery. Some $T$ cell lines recovered from the autoimmune infiltrate were found to be encephalitogenic.

\section{Methods}

Rats. Inbred Lewis rats were supplied by the Animal Breeding Center of this Institute and were used at 2-3 mo of age. Rats were matched for age and sex in each experiment.

Antigens. Mycobacterium tuberculosis (MT) H37Ra was purchased from Difco Laboratories, Inc., Detroit, MI. BP was prepared as de- 
scribed (3) from the spinal cords of guinea pigs, without the step of purification by column chromatography. Cloned recombinant hsp65 antigen and hsp 70 were produced at the National Institute of Public Health and Environmental Protection, Bilthoven, The Netherlands, and kindly supplied by Dr. Ruurd van der Zee (9). Peptides of myelin BP were synthesized according to published sequences of the protein.

Induction of EAE. The disease was induced by injecting both hind foot pads with $0.05 \mathrm{ml}$ containing $25 \mu \mathrm{g}$ of BP and $200 \mu \mathrm{g}$ of MT emulsified in equal volumes of incomplete Freund's adjuvant and PBS. Passive EAE was adoptively transferred by intraperitoneal injection of $10^{7} \mathrm{BP}$-activated cells of the D9 encephalitogenic clone. Clinical EAE was observed in over $90 \%$ of immunized rats $10-12 \mathrm{~d}$ after BP/CFA induction and $5 \mathrm{~d}$ after clone injection. Severity of disease was graded as follows: +1 , paralysis of tail; +2 , paralysis of hind legs; +3 , paralysis extending to the thoracic spine; +4 , moribund state.

Preparation of cell suspensions. At various intervals after induction of EAE, rats were killed by anesthesia with ether. Cell suspensions were prepared from lymph nodes, spleens, or thymuses by pressing the organs through a fine wire mesh. Peripheral blood lymphocytes were collected using a Ficoll hypaque gradient (Pharmacia, Uppsala, Sweden). Spinal cords were extruded from the vertebral column aseptically by passing a wooden applicator through the spinal canal. Spinal cord lymphocytes were obtained by gentle grinding of spinal cord tissue with a 15-ml Dounce tissue grinder (model 357544, Wheaton Industries, Millville, NJ) in $10 \mathrm{ml}$ of PBS. The homegenate was then subjected to two or three cycles of Ficoll gradient separation. The nervous tissue remained at the interface and the lymphocytes were recovered from the pellet. Cells representing the meningeal compartment were recovered by washing the surface of the brain with PBS, as described (12).

Limiting dilution analysis (LDA). The lymphocytes obtained from the various sources were seeded in 96 -well, round-bottom microtiter plates at decreasing concentrations from $4 \times 10^{3}$ cells per well to 1 cell per well, in doubling dilutions. (Numbers of cells $>4 \times 10^{3}$ per well produced multiple hit curves; see below.) The cells were seeded in 200 $\mu \mathrm{l}$ of Dulbecco's modified Eagle's medium supplemented with 2 mercaptoethanol $\left(5 \times 10^{-5} \mathrm{M}\right)$, L-glutamine $(2 \mathrm{mM})$, sodium pyruvate (1 $\mathrm{mM})$, penicillin $(100 \mu / \mathrm{ml})$, streptomycin $(100 \mu \mathrm{g} / \mathrm{ml})$, nonessential amino acids ( $1 \mathrm{ml} / 100 \mathrm{ml}$, Bio Lab, Jerusalem, Israel), fetal calf serum $10 \%$ (vol/vol), and T cell growth factors (TCGF) from Con A supernatant of autologous splenocytes (10\% vol/vol). In preliminary experiments we found that the optimal time for adding TCGF was day 0 : adding TCGF on day 0 resulted in frequencies 10 -fold higher than in those cultures to which TCGF was added on day 4. Irradiated thymocytes $(2,500 \mathrm{rad})$ were added as feeder cells $\left(10^{5}\right.$ per well). Cultures were performed in the absence or presence of antigens $(10 \mu \mathrm{g} / \mathrm{ml})$, Con $A$ $(1.2 \mu \mathrm{g} / \mathrm{ml})$, or irradiated $(2,500 \mathrm{rad}) 10^{5}$ allogeneic BN spleen cells. 24 wells were seeded at each cell concentration: 20 wells with antigen and 4 wells as controls without antigen. Plates were incubated for $7 \mathrm{~d}$ in $7 \%$ $\mathrm{CO}_{2}$ at $37^{\circ} \mathrm{C}$. The uptake of ${ }^{3}[\mathrm{H}]$ thymidine was measured in the last 18 $\mathrm{h}$ of culture. In addition to measuring thymidine incorporation, each well was observed on day 7 for cell growth. A positive well was scored by a visible cellular aggregation and thymidine uptake exceeding the mean plus $2 \mathrm{SD}$ of the background wells cultured without antigens, Con A, or allogeneic spleen cells. Percent negative wells was plotted against cell concentration on a semilog plot. The cell number corresponding to $37 \%$ negative wells was considered to represent the frequency of the $T$ cells responding to the antigen (13).

$T$ cell lines. Antigen-specific T cell lines were established from wells showing positive growth on duplicate plates containing identical numbers of cells per well as the LDA plates. Positive wells containing 250 1,000 cells per well were individually expanded by repeated stimulation with specific antigen and antigen presenting cells every 10-12 d. The stimulation was performed in the same medium as that used for the LDA test, without the fetal calf serum and the Con A supernatant, and with $1 \%$ autologous Lewis serum. BP and MT were added to the wells at $10 \mu \mathrm{g} / \mathrm{ml}$. After stimulation for $3 \mathrm{~d}$, the cells were collected, washed, and cultured for a resting phase for 5-9 d in resting medium (identical to LDA medium but without antigens).
$T$ cell proliferation assay. When $T$ cell lines reached adequate numbers (after the fourth or fifth stimulation) at the end of a rest phase, $10^{5}$ line cells were seeded in 96 round bottom microtiter wells (Greiner Labortechnik, Nürtingen, FRG) with $5 \times 10^{5}$ irradiated (2500 rad) thymocytes as accessory cells. BP and MT were added at $10 \mu \mathrm{g} / \mathrm{ml}$; hsp65, hsp70, and BP peptides were added at $5 \mu \mathrm{g} / \mathrm{ml}$. The proliferation was performed in stimulation medium as described above. The cultures were incubated in triplicate for $72 \mathrm{~h}$ at $37^{\circ} \mathrm{C}$ in humidified air containing $7 \% \mathrm{CO}_{2}$. Each well was pulsed with $1 \mu \mathrm{Ci}$ of $\left[{ }^{3} \mathrm{H}\right]$ thymidine (10 ci/mmol sp act; Nuclear Research, Negev, Israel) for the final $18 \mathrm{~h}$. The cultures were then harvested on fiberglass filters and the proliferative response expressed as counts per minute (CPM).

Encephalitogenicity. Anti-BP T cell lines were injected intraperitoneally, $2 \times 10^{7}$ cells per rat to groups of five rats and the rats were observed daily for the clinical signs of EAE. Line mediated EAE appeared on days 4-6 postinjection and lasted 2-4 d.

Flow cytometry. Line cells were stained at $4^{\circ} \mathrm{C}$ for $45 \mathrm{~min}$. with the following monoclonal antibodies at a 1:100 dilution: w3/25 for CD4 MRC ox-8 for CD8, and R7.3 for $\alpha \beta$ TCR. All antibodies were purchased from Serotec, Oxford, UK. Secondary rabbit anti-mouse FITC conjugated antibodies were used at a $1: 50$ dilution at $4^{\circ} \mathrm{C}$ for $30 \mathrm{~min}$. The cells were then washed and fluorescence was measured using the FACS 440 (Becton Dickinson \& Co., Mountain View, CA).

\section{Results}

Frequencies of $T$ cells in naive rats. It has been shown that encephalitogenic anti-BP T cells may be recovered from naive Lewis rats (14). Naive rats would also be expected to have $T$ cells responsive to MT and hsp antigens (15). It was of interest therefore to determine the frequencies of these $T$ cells in naive rats before the induction of EAE.

LDA assays for naive anti-BP T cells were done using cell concentrations of up to $2 \times 10^{5}$ lymph node cells per well. Although some wells were positive, the results tended to be inconsistent (not shown). However, Fig. 1 shows that the LDA assay could detect the frequencies of $T$ cells reactive to $\mathrm{MT}$, hsp65, and hsp70, which were in the range of $1: 5,000$ to $1: 15,000$

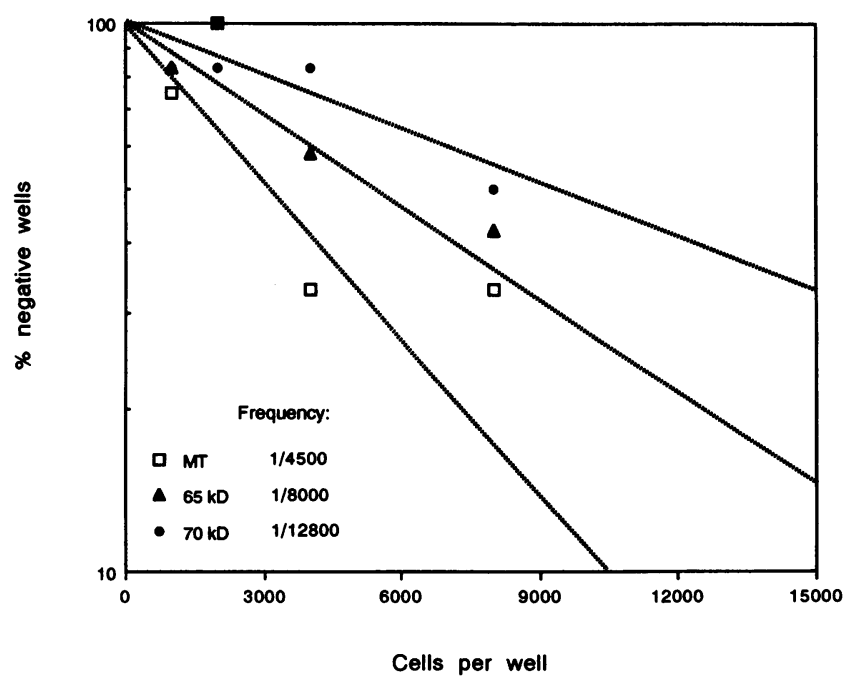

Figure 1. LDA of the frequency of T cells responding to Mycobacterium tuberculosis $(M T, \square)$, hsp $65(65 \mathrm{kD}, \Delta)$, and hsp $70(70 \mathrm{kD}, \bullet)$, from lymph node lymphocytes of naive Lewis rats. The percent negative wells was plotted as a function of cells seeded per well, in a semilogarithmic curve. 


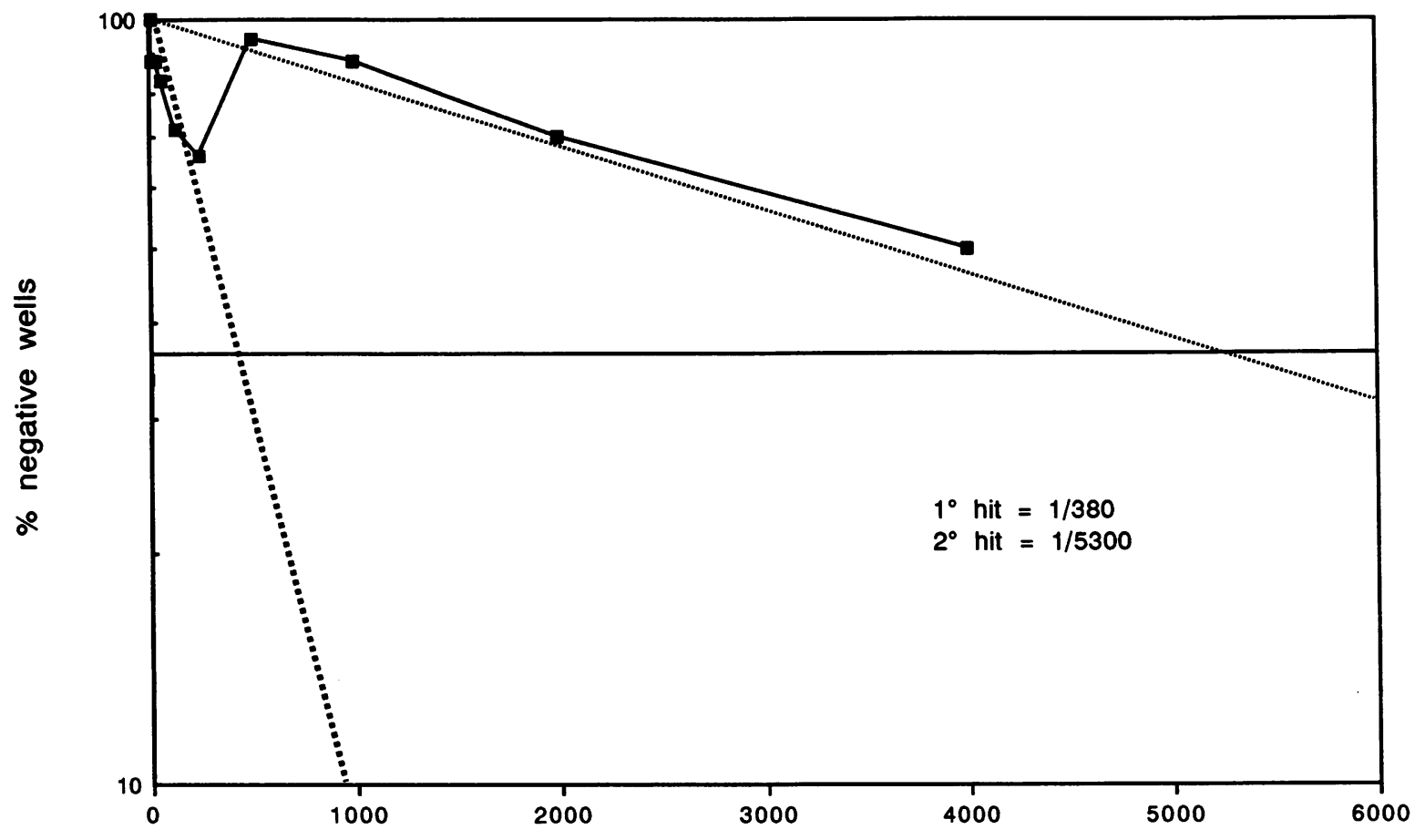

Cells per well

Figure 2. LDA of the frequency of T cells responding to BP of lymphocytes isolated from the draining popliteal lymph nodes, on day 15 of EAE induction. The curve shows a "multihit" pattern; the first hit frequency of BP responsive cells is 1:380 and the second hit frequency is 1:5,300.

Multiple-hit frequencies of anti-BP T cells. Despite the indeterminacy of the frequency of anti-BP T cells in naive rats, induction of EAE led to appreciable results. Note in Fig. 2 that the LDA assay showed a multiple-hit response yielding two frequencies: the first at cell concentrations $<10^{3}$ per well and the second at cell concentrations $>10^{3}$ per well. Multiple-hit curves in LDA assays have been explained by the presence of regulatory cells that may be present and suppress $T$ cell reactivity at higher cell concentrations (16). The presence of regulatory cells in naive rats (16) could also explain the difficulty in establishing the frequency of anti-BP T cells before immunization described above. As we were interested in measuring the highest frequencies of $T$ cells, we focused on the first hit and carried out most of our experiments using concentrations of cells of $4 \times 10^{3}$ and less per well.

Enrichment of BP-responsive T cells in EAE. Fig. 3 shows the results of the LDA of T cells isolated at the onset of EAE (day 12 after immunization) from the draining popliteal lymph nodes, the spleen, the peripheral blood, and the spinal cord tissue. The frequency of anti-BP T cells in the spinal cord was 10 times higher than that in the draining lymph nodes, and about 50 times higher than that in the spleen and peripheral blood. Before the onset of EAE, on day 9, we did not find cells in the spinal cord (not shown).

Frequencies of $T$ cells responsive to $M T$ or to alloantigens. Fig. 4 and 5 show the results of the LDA to BP, to MT, and to BN spleen cells on day 12 after immunization with BP/CFA. The frequencies from the spinal cord are compared to those from the draining popliteal lymph nodes. The frequency of MT reactive cells in the lymph nodes was higher than the frequency of $B P$ reactive $T$ cells, whereas in the spinal cord the BP reactive $\mathrm{T}$ cells were more common (Fig. 4). Nevertheless, there was a relative increase in the frequency of MT-reactive $\mathrm{T}$ cells in the spinal cord compared to the draining lymph nodes (26:43). The difference is even more marked if we consider the fact that the frequency of $\mathrm{T}$ cells responsive to Con $\mathrm{A}$ (the maximum number of $T$ cells detectable by proliferation) was lower in the spinal cord than in the lymph nodes (2:1).

Fig. 5 shows that the spinal cord infiltrate contained alloreactive $\mathrm{T}$ cells in a frequency similar to that found in the periph-

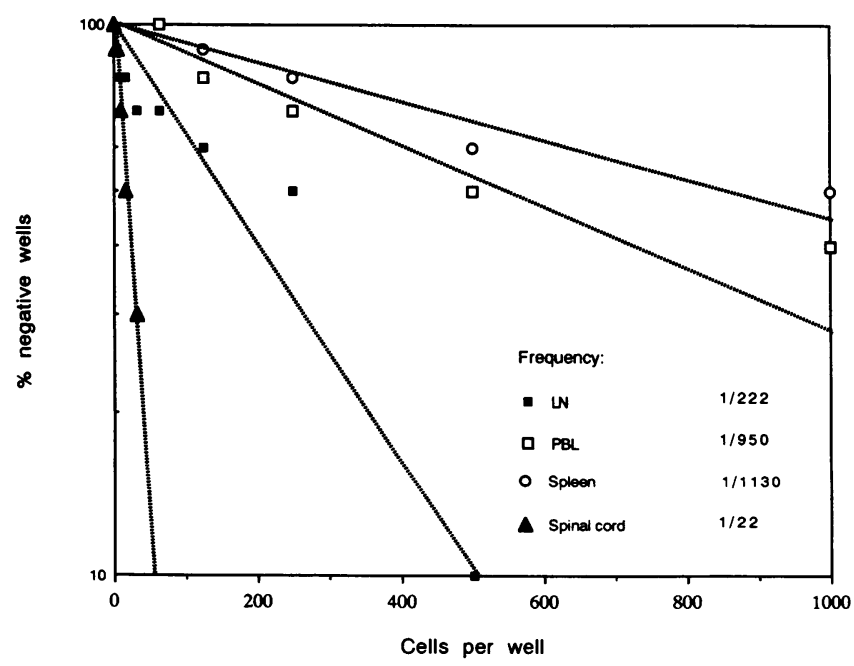

Figure 3. LDA of the frequency of T cells responding to BP of lymphocytes isolated from the draining popliteal lymph nodes $(L N, \varpi)$, peripheral blood lymphocytes $(P B L, \square)$, spleen $(0)$, and spinal cord $(\Delta)$, on day 12 of EAE induction. The rats showed a clinical score 3 or 4 on the day of analysis. 

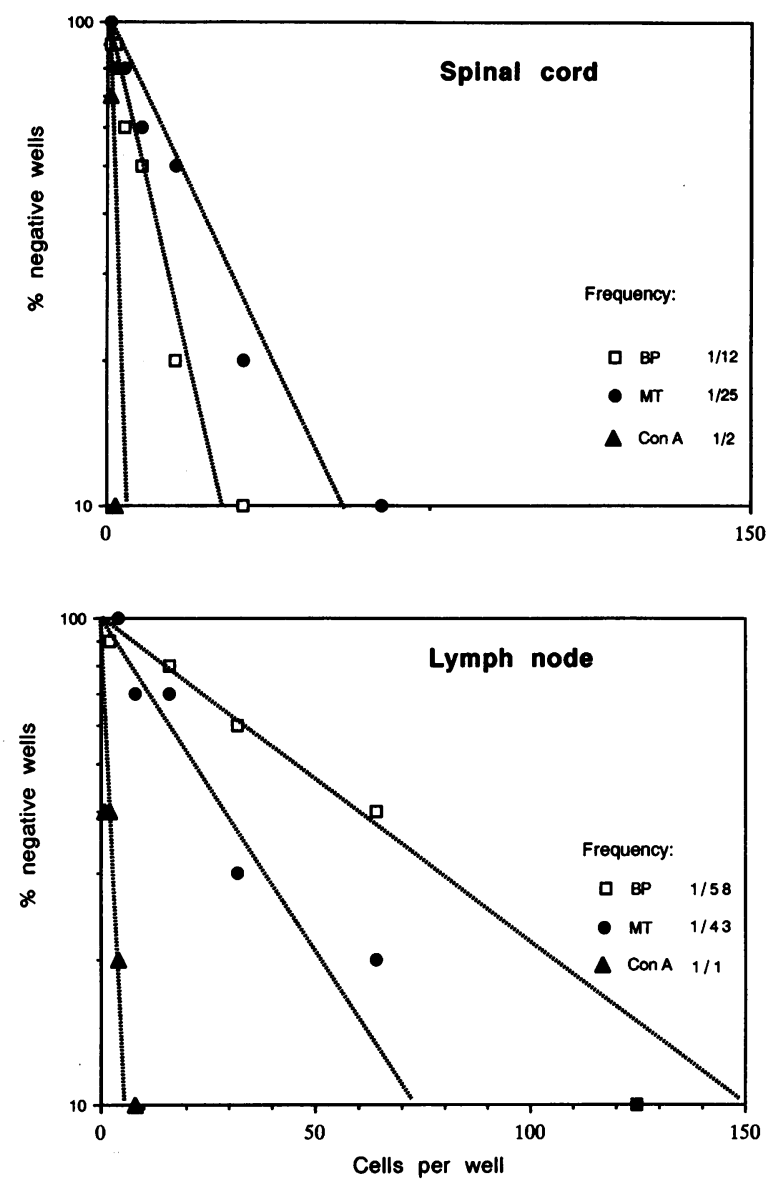

Figure 4. Comparison of LDA results of lymphocytes isolated from the spinal cord to those isolated from the draining popliteal lymph node obtained on day 12 of EAE induction. We determined the fre-

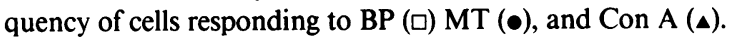

ery. Studies examining the frequency of alloreactive $\mathrm{T}$ cells in mice yielded similarly high frequencies (17). Thus, the spinal cord lesions of EAE appear to accumulate T cells differentially: the lesions are greatly enriched for BP-reactive $\mathrm{T}$ cells, somewhat enriched for MT reactive $\mathrm{T}$ cells, and not enriched for alloreactive $\mathrm{T}$ cells.

$T$ cell frequencies in the meningeal surface and the spinal cord. The cerebrospinal fluid (CSF) compartment is readily accessible in patients with inflammatory and autoimmune diseases of the CNS, but the CNS tissue itself is not. It was therefore of interest to compare the $\mathrm{T}$ cells present in the meningeal surface with those isolated from the parenchyma of the spinal cord. As the CSF bathes the surface of the brain, it was possible to obtain CSF lymphocytes by washing the brain in a way that disrupted the meninges. Analysis of cell surface markers and subtypes has shown that cells isolated from the brain surface were similar to cells obtained from the EAE lesion (12). Here we compared the frequencies of $\mathrm{T}$ cells responsive to $\mathrm{BP}, \mathrm{MT}$, and Con A from both compartments. Fig. 6 shows the results of an LDA performed on day 15 after immunization. The results indicate that the meningeal compartment reflects accurately the numbers of BP and $M T$ reactive $T$ cells present in the spinal cord itself during EAE.

Kinetics of BP and MT frequencies. We examined the frequencies of cells responding to BP and MT in days 15 and 29 following EAE induction and compared them to day 12. As
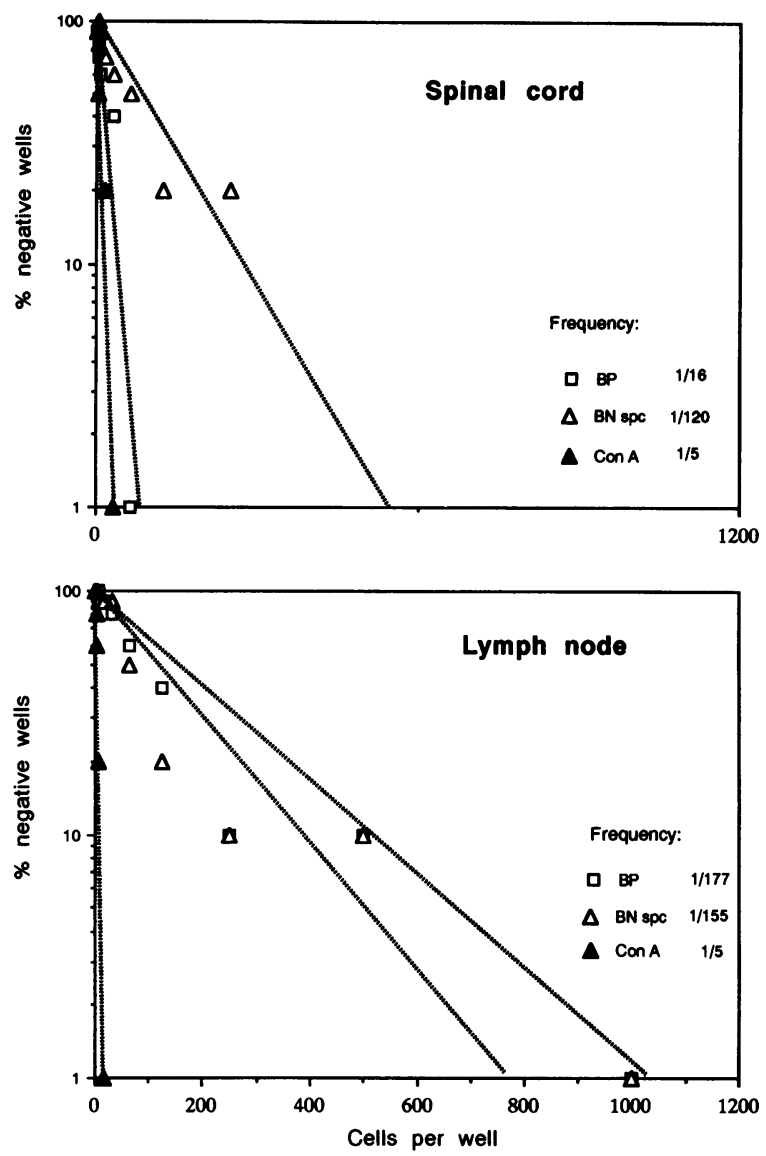

Figure 5. Comparison of LDA results of lymphocytes isolated from the spinal cord to those isolated from the draining popliteal lymph node obtained on day 15 of EAE induction. We determined the frequency of cells responding to BP ( $\square$ ), allogeneic irradiated BrownNorway spleen cells $(B N s p c, \Delta)$, and Con $\mathrm{A}(\boldsymbol{\Delta})$.

can be seen from Fig. 7, BP and MT cells were detected both in the lesion and in the periphery through day 29 of disease induction. The frequency of BP cells was higher in the spinal cord during the paralytic phase of the disease whereas following recovery it was similar to that found in the periphery (Fig. 7).

Encephalitogenic $T$ cell lines. In searching the literature we found only one study of BP-reactive $T$ cell lines grown from the spinal cords of animals with EAE (18). None of the lines was reported to transfer EAE, and only one was found to cause inflammation in the CNS. Table I shows the characteristics of the 10 lines we isolated from the spinal cord on day 12 after induction of EAE. The majority of the lines ( 9 of 10$)$ responded to the major encephalitogenic peptide in Lewis rats, 71-90, two of these lines also responded to another peptide epitope, 1-11. All of the lines responding only to the 71-90 epitope were found to be encephalitogenic. The lines with specificity to both 71-90 and 1-11 were not found to mediate EAE. Line 8 responded to whole BP but did not respond to either of the two peptide epitopes and was not encephalitogenic. All of the lines expressed the $\alpha \beta$ T cell receptor and were $\mathrm{CD}^{+}$and $\mathrm{CD} 8^{-}$. Fig. 8 shows the results of the proliferation assay of the various anti-BP lines. Type 1 lines responded to the 71-90 peptide only and were encephalitogenic; type 2 lines responded both to the 1-11 peptide and to the 71-90 peptide and were not encephalitogenic; the type 3 line, line 8 , did not respond to either of 

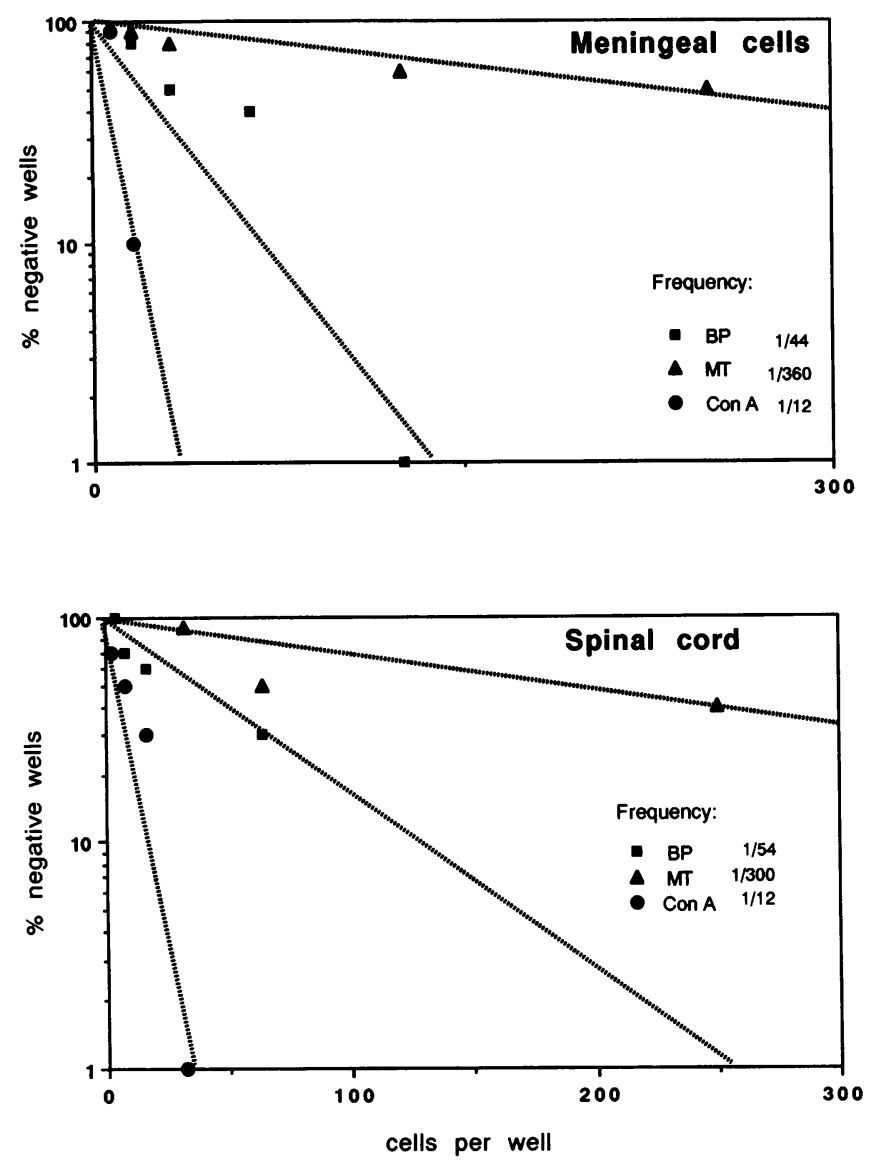

Figure 6. Comparison of LDA results of lymphocytes isolated from the spinal cord to those isolated from the brain surface (meninges) obtained on day 15 of EAE induction. We determined the frequency

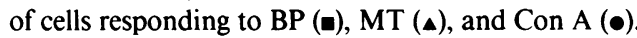

the peptides and was not encephalitogenic. None of the antiBP lines responded to MT.

$T$ cell lines reactive to hsp 65 and $h s p 70$. From positive wells containing 500 cells, we developed lines to MT, and tested them after the fourth stimulation for reactivity to various antigens. Fig. 9 shows the proliferation profile of six such lines. All six lines responded to hsp 65 and two of the lines also prolifer-

Table I. Pathogenicity and FACS Analysis

\begin{tabular}{|c|c|c|c|c|c|}
\hline \multirow[b]{2}{*}{ Line No. } & \multicolumn{3}{|c|}{ BP recognition } & \multirow{2}{*}{$\begin{array}{l}\text { Pathogenicity } \\
\left(2 \cdot 10^{7} \text { cells }\right)\end{array}$} & \multirow[b]{2}{*}{ FACS } \\
\hline & $71-90$ & $1-9$ & Other & & \\
\hline 6 & + & - & - & ND & $\alpha \beta^{+} \mathrm{CD}^{+} \mathrm{CD} 8^{-}$ \\
\hline 7 & + & - & - & + & ND \\
\hline 8 & - & - & + & - & $\alpha \beta^{+} \mathrm{CD}^{+} \mathrm{CD}^{-}$ \\
\hline 11 & + & - & - & + & $\alpha \beta^{+} \mathrm{CD}^{+} \mathrm{CD}^{-}$ \\
\hline 12 & + & - & - & ++ & ND \\
\hline 14 & + & - & - & +++ & $\alpha \beta^{+} \mathrm{CD}^{+} \mathrm{CD}^{-}$ \\
\hline 18 & + & - & - & +++ & $\alpha \beta^{+} \mathrm{CD} 4^{+} \mathrm{CD} 8^{-}$ \\
\hline 21 & + & + & - & - & ND \\
\hline 22 & + & + & - & - & $\alpha \beta^{+} \mathrm{CD}^{+} \mathrm{CD}^{-}$ \\
\hline 23 & + & - & - & +++ & $\alpha \beta^{+} \mathrm{CD} 4^{+} \mathrm{CD} 8^{-}$ \\
\hline
\end{tabular}

ND, not determined.
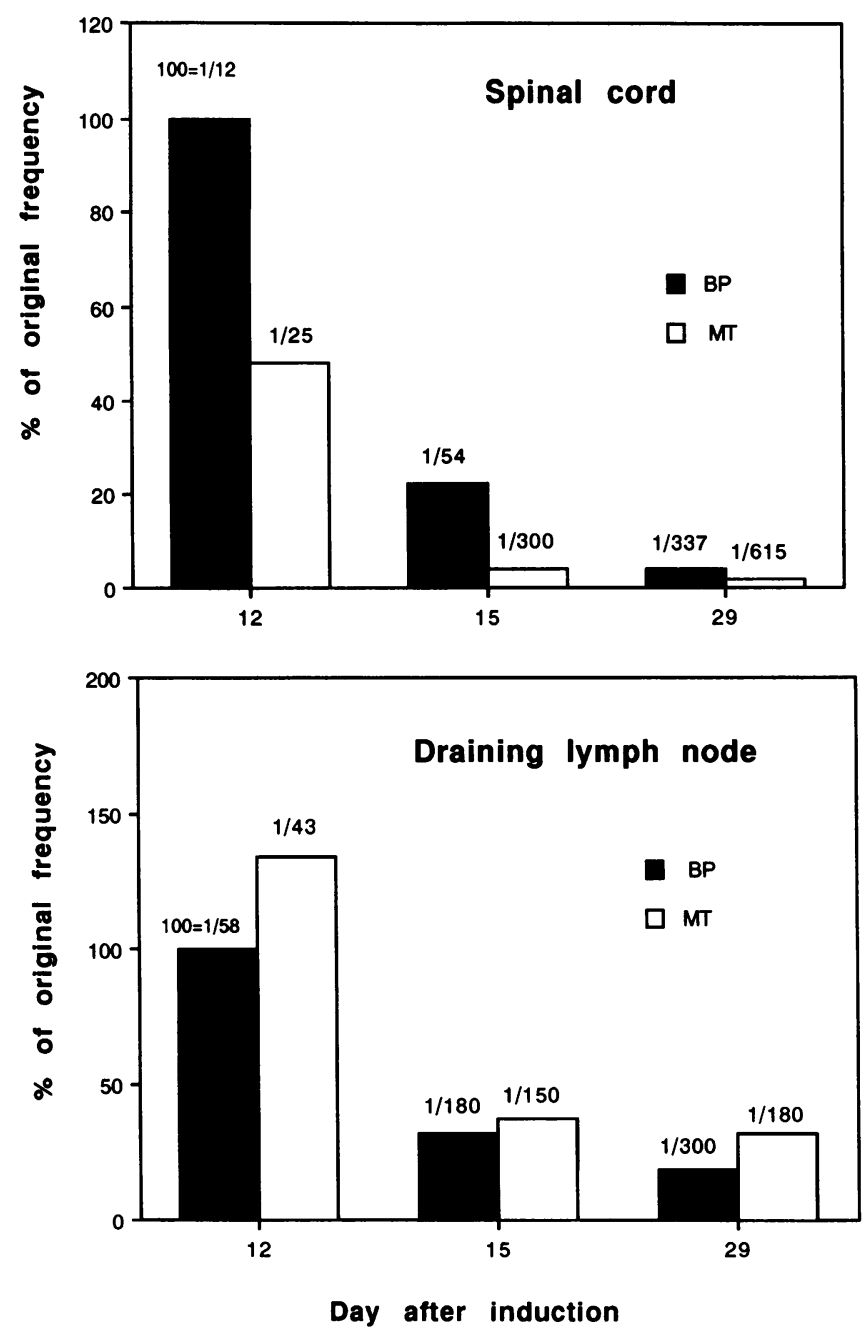

Figure 7. LDA results from spinal cord as compared to lymph nodes on days 12,15, and 29 after EAE induction. The frequency of cells responding to $\mathrm{BP}$ on day 12 was arbitrarily chosen to be $100 \%$, the frequency of cells responding to MT on day 12 , as well as the frequencies to BP and MT on days 15 and 29 were calculated relative to the $\mathrm{BP}$ frequency on day 12 . The actual frequencies are given above each column.

ated in response to hsp70. Mapping of the segment of hsp65 recognized by the $\mathrm{T}$ cell lines was done using deletion mutants of the molecule (8). The lines all responded to an area of hsp65 composed of residues 171 to 276 (not shown). Within this segment of hsp65, there was no response to the 180-188 peptide recognized by arthritogenic rat $\mathrm{T}$ clone $\mathrm{A} 2 \mathrm{~b}(8)$ or to the other available peptide, 227-236 (not shown). Fig. 10 shows an LDA assay of $T$ cells responsive to hsp65 which confirms the high frequency of such cells.

Frequency of HSP 65 reactive $T$ cells in passive EAE. To investigate whether the enrichment of hsp 65 reactive $T$ cells in the lesion resulted from activation of such $\mathrm{T}$ cells by the MT in the adjuvant or whether the inflammatory lesion itself attracted anti-hsp65 T cells, we investigated EAE produced by adoptive transfer of an encephalitogenic clone D9. As shown in Fig. 11, the frequency of T cells responding to hsp65 was 30fold higher in the spinal cord infiltrate as compared to the peripheral lymph nodes. Thus, the accumulation of anti-hsp $65 \mathrm{~T}$ cells did not require recent active immunization to hsp 65 in the 


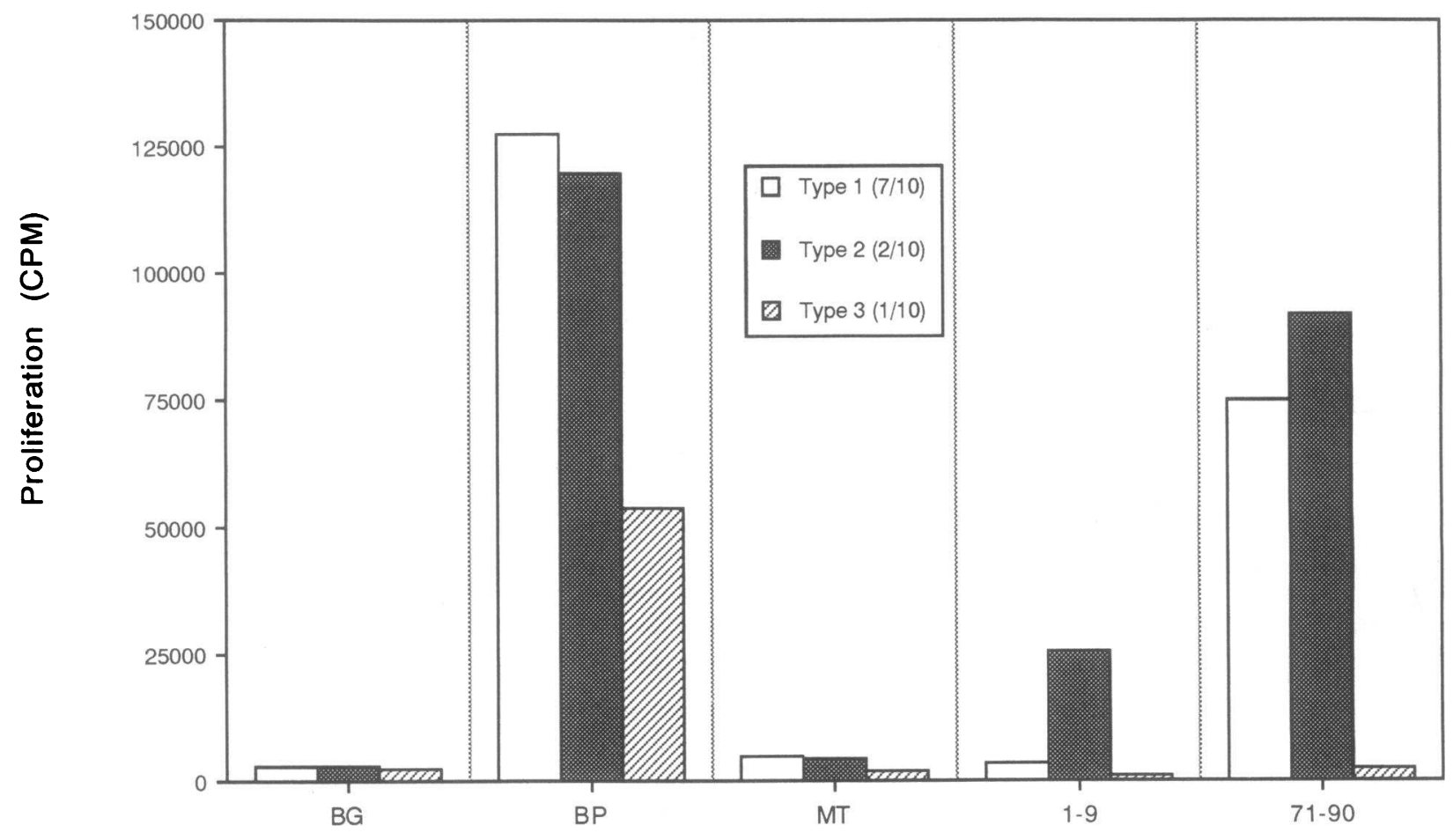

\section{Antigen:}

Figure 8. T cell proliferation profile of $10 \mathrm{BP}$-specific lines derived from the spinal cord LDA cultures, established on day 12 of EAE induction. Proliferation was determined by thymidine incorporation in response to BP, MT, and BP peptides 1-9, 71-90, 84-101, and 89-100. Seven lines (type 1) showed a proliferative response to BP and BP peptide 71-90, two lines responded to BP, BP peptide 71-90 and a smaller response to peptide 1-9 (type 2), and one line responded to BP but did not proliferate to any of the peptides examined (type 3). No lines responded to peptides $84-101$ or $89-100$.

adjuvant. Interestingly, the frequency of BP responding $\mathrm{T}$ cells was lower in the spinal cord of passive EAE relative to the frequency in actively induced disease.

\section{Discussion}

In the present study we have analyzed the $T$ cell composition of the autoimmune infiltrate in EAE by LDA and by the establishment of T cell lines. Our results indicate that BP-reactive T cells are markedly enriched in the lesion at the peak of disease with a decline in frequency after recovery. The BP reactive cells were expanded to $T$ cell lines, and were found to respond mainly to the major encephalitogenic epitope in the lewis rat (residues 71-90); however, the responses were not restricted to this epitope. We also found that $\mathrm{T}$ cell lines originating from the CNS were pathogenic and caused EAE in naive recipients. Interestingly, the meningeal cells were found to be similar to the spinal cord tissue in the frequencies of BP-and MT-reactive $\mathrm{T}$ cells. Thus the meningeal cells can provide information about $T$ cells present in the CNS tissue, perhaps because meningitis is a prominent feature of EAE (7). Indeed much of the inflammatory infiltrate in the CNS in EAE is perivascular and therefore may be in direct contact with the meninges (7).

The frequencies of BP reactive $T$ cells we found are higher than those reported previously $(7,19,20)$. Other investigators may have missed the high frequency of reactive $T$ cells because they did not test concentrations of cells $<800-1,000$ per well
$(19,20)$. As we have shown here, the LDA assay of responsiveness to BP shows a multihit pattern (Fig. 2); consequently the higher frequencies would be missed by failing to test low concentrations of cells. It is also possible that our cultures were more conducive to the proliferation of low numbers of responding $T$ cells because we enriched the medium with growth factors from the onset, while others added IL-2 only on day 4 (21). It should be noted that the LDA can be influenced greatly by differences in culture conditions. We found that adding TCGF from start of the culture increased the sensitivity of the test 10 -fold. Indeed, various studies of the frequencies of alloreactive cytolytic $T$ cells have shown large variations in frequency (17). In agreement with the high frequencies found in this study, analysis of the T cell precursor frequency to $\mathrm{Myco}$ bacterium leprae antigens in patients with leprosy, revealed a 100-fold enrichment in the lesion as compared to the blood; a frequency of 1:48 in the lesion and 1:4,232 in blood (22). Moreover, $85 \%$ of $\mathrm{T}$ cell clones raised from the thyroid of a patient with Graves' disease using a non-antigen-specific method were found to react to thyroidal autoantigens (23).

To verify that the positive wells actually contained BP-specific $T$ cells, we raised $T$ cell lines from these wells by repeated stimulation with the antigen. In our experience, anti-BP T cell lines were readily established even when the number of cells seeded per well was as low as 250 . These lines were shown to have specific reactivity to BP and cause EAE (Table I). The fact that antigen-specific $T$ cell lines could be isolated in this man- 


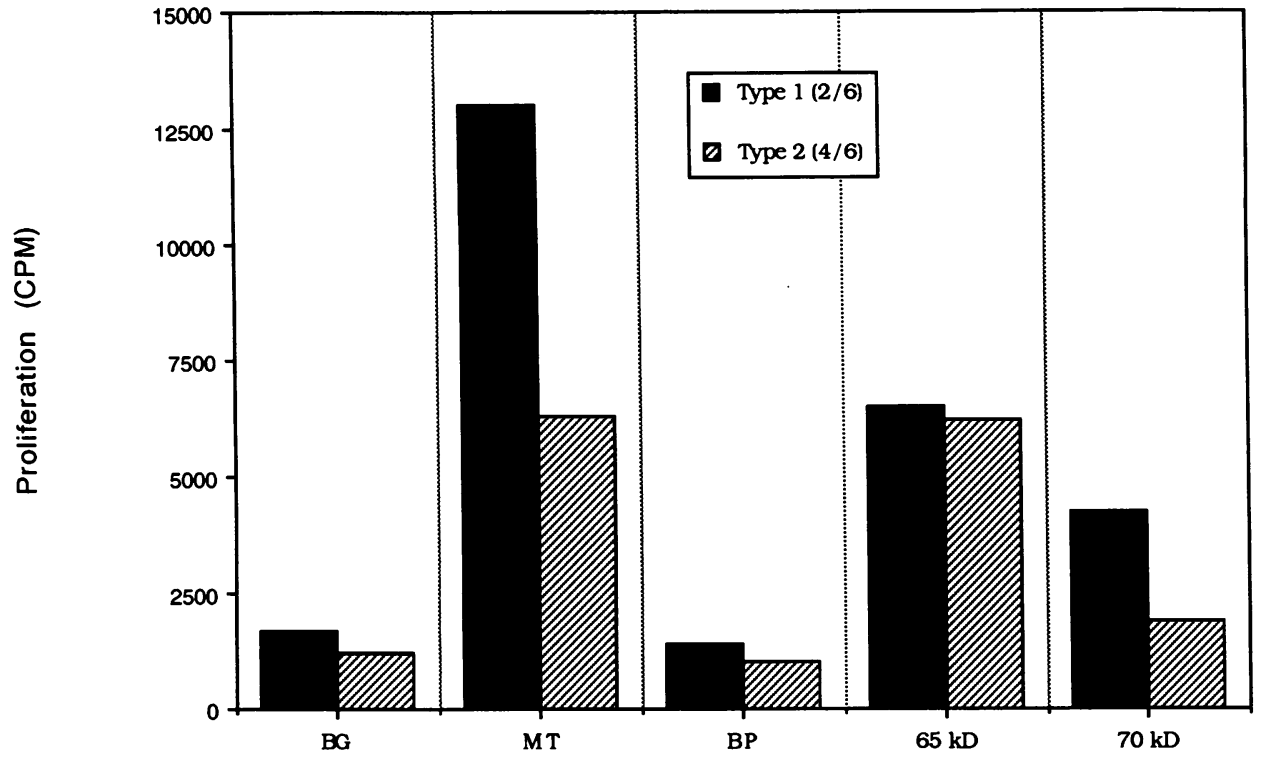

Antigen:
Figure 9. T cell proliferation profile of $6 \mathrm{MT}$ specific lines derived from the spinal cord LDA cultures, established on day 15 of EAE induction. Proliferation was determined by thymidine incorporation in response to BP, MT, 65- and 70-kD hsp. ner indicates that the frequency of $1: 2,000$ to 41,700 reported in the literature $(7,19,20)$ is certainly an underestimation of the true frequency.

In addition to a high frequency of BP-specific T cells, the spinal cord and meningeal membranes contained a high frequency of $\mathrm{T}$ cells responsive to $\mathrm{MT}$. The specificity of the antiMT T cells was shown to be largely directed to hsp65 and to hsp70. It was reported that $20 \%$ of the anti-MT T cells in mice immunized to MT responded to hsp65 (21) indicating the hsp65 is immunologically dominant. However, our finding that all six anti-MT T cell lines contained reactivity to hsp65 indicates that such $T$ cells may selectively accumulate in the EAE lesion. Res et al. (24) found anti-hsp65 T cells in plural effusions and suggested that such cells might be attracted to

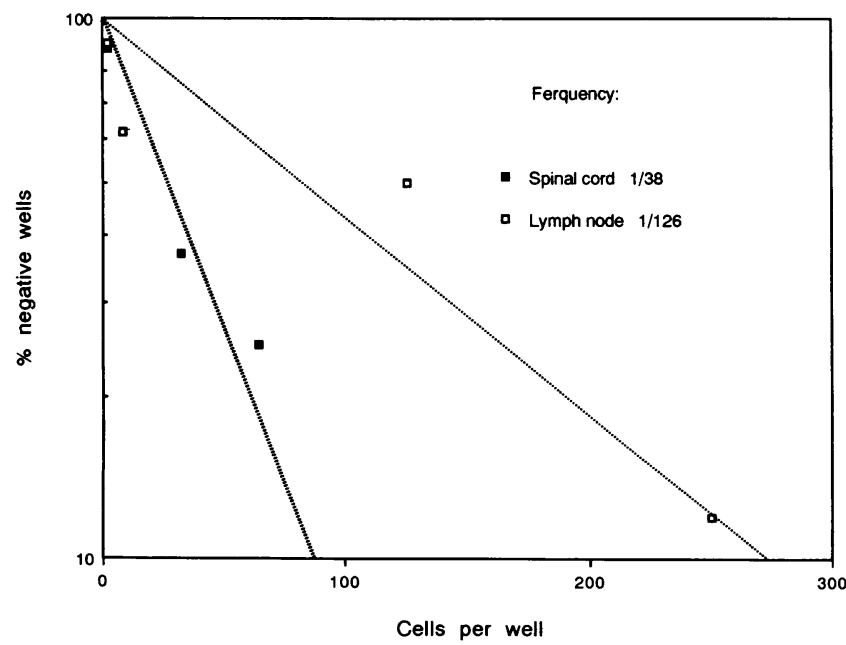

Figure 10. Comparison of LDA results of lymphocytes isolated from the spinal cord to those isolated from the draining popliteal lymph node obtained on day 15 of EAE induction, to the hsp 65 .
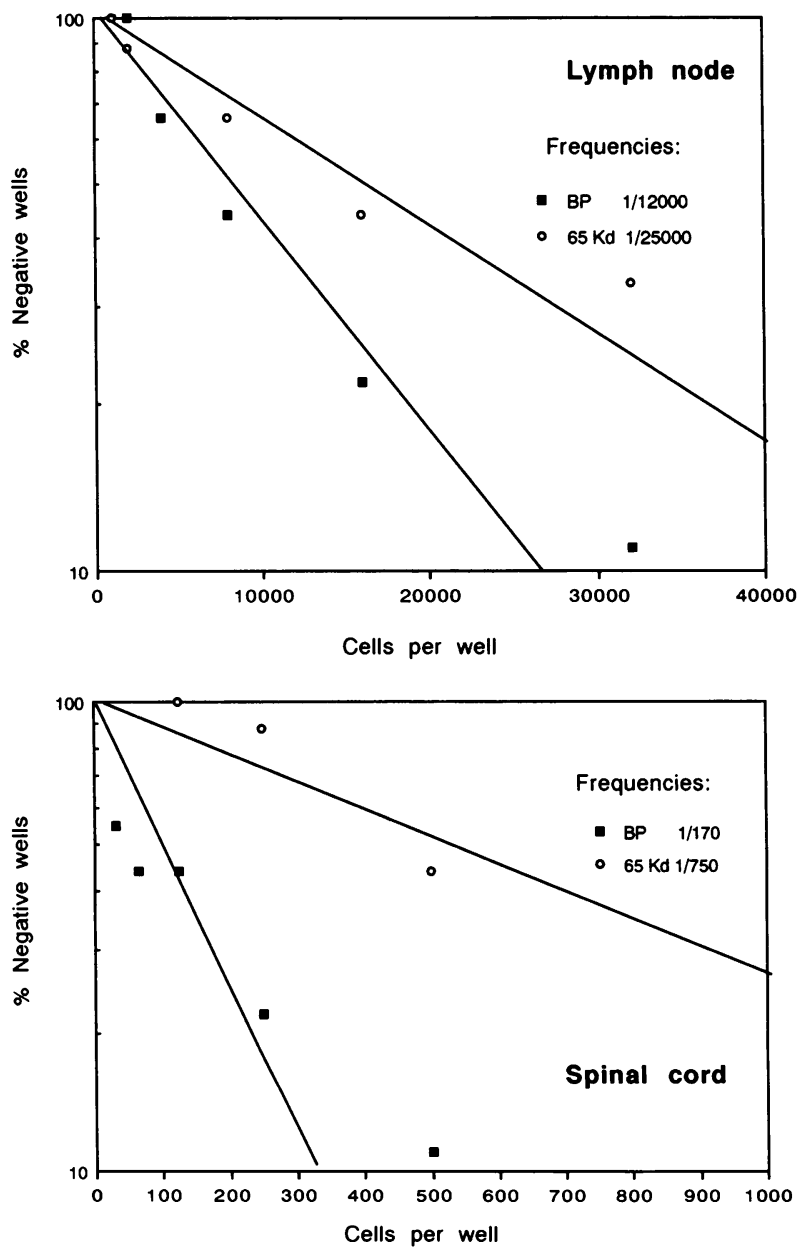

Figure 11. Comparison of LDA results of lymphocytes isolated from the spinal cord to those isolated from popliteal lymph nodes on day 6 of induction of passively transferred EAE by the encephalitogenic clone D9. LDA was performed to BP- and hsp65-reactive T cells. 
sites of inflammation produced by any cause. Activated macrophages express augmented amounts of hsp65 (25) and it is quite likely that endogenous hsp65 accompanies the stress of inflammation (26). As shown here, even naive rats have an appreciable frequency of anti-hsp65 T cells (Fig. 1) and it is conceivable that the accumulation of these cells at sites of inflammation might serve to amplify beneficial immune responses in infections (27).

The finding that hsp65-reactive cells are enriched in the spinal cord of EAE rats can be interpreted in two ways: These cells are activated and are therefore capable of entering the lesion nonspecifically. However, nonspecific $\mathrm{T}$ cell migration was found by Hickey et al. (8) to be transient, so it could not fully explain the presence of these cells even after $29 \mathrm{~d}$ of EAE induction. Alternatively, the anti-hsp65 $\mathrm{T}$ cells could have crossed the blood brain barrier due to their state of activation, but their continued presence in the lesion might have been due to local presentation of hsp65 in the central nervous system. Indeed recent work in multiple sclerosis has revealed both $\gamma \delta \mathrm{T}$ cells and local hsp65 expression in chronic plaques from brain tissue (28). The increase in frequency of hsp65 $\mathrm{T}$ cells in the lesion in passive EAE (Fig. 11) favors the hypothesis that the accumulation of these cells in the spinal cord is not merely due to their recent activation, and suggests a role for these cells in the inflammatory process.

In addition to the accumulation of hsp 65 reactive $T$ cells in inflammatory lesions in general $(24)$, hsp65 reactive $T$ cells have been shown to be specifically associated with adjuvant arthritis in Lewis rats (9) and with the autoimmune diabetes of nonobese diabetic strain mice $(10,11)$. The anti-hsp65 T cells isolated from the lesions of EAE did not react to the hsp65 epitopes associated with arthritis (180-188) or with diabetes (424-447). Further work is required to map the hsp65 epitope recognized by the $T$ cells in EAE lesions to determine whether the cells are truly autoimmune and what the significance of these $T$ cells might be. Although hsp65 reactive $T$ cells were found to be enriched for $\gamma \delta \mathrm{T}$ cells (29), the anti-hsp65 T cell lines derived from the spinal cord, similar to the anti-BP T cells, express $\alpha \beta \mathrm{T}$ cell receptors and are $\mathrm{CD}^{+}, \mathrm{CD}^{-}$(not shown).

Despite the early work that pointed to the nonspecific nature of autoimmune infiltrates (4), many investigators of autoimmune diseases have attempted to obtain autoantigen-specific $T$ cells by culturing $T$ cells from the lesion using nonspecific mitogens, synovia or synovial fluid in rheumatoid arthritis (30), pancreas in murine diabetes (31), or CSF in multiple sclerosis (32). Indeed oligoclonal T cells were found in these compartments in both multiple sclerosis and rheumatoid arthritis $(30,32)$.

If one considers EAE as a model for human autoimmune disease, then the results of our study have several implications for the design of studies in humans. The lesion and the compartment surrounding it are a rich source of autoantigen specific effector $T$ cells. Nevertheless, the $T$ cells responsible for causing the disease are still a minority of the $T$ cells that can respond to mitogens. Thus, without the confirmation of the specific antigen, it should not be assumed that predominant $T$ clones are causally related to the disease. Finally, specific autoimmune $T$ cells may persist in the tissues after clinical recovery. How these autoimmune $\mathrm{T}$ cells are silenced and whether they are the source of relapses remain to be determined.

\section{Acknowledgments}

Dr. Mor was supported by the Chief Scientist's Office, Ministry of Health, Israel; Dr. Cohen is the incumbent of the Mauerberger Chair of Immunology.

\section{References}

1. Paterson, P. Y., and R. H. Swanborg. 1988. Demyelinating diseases of the central and peripheral nervous systems. In Immunological Diseases, 4th edition. M. Samter, editor. Little Brown \& Co., Boston. 1877 pp.

2. Naparstek, Y., A. Ben-Nun, J. Holoshitz, T. Reshef, A. Frenkel, M. Rosenberg, and I. R. Cohen. 1983. T lymphocyte lines producing or vaccinating against autoimmune encephalomyelitis (EAE): functional activation induces peanut agglutinin receptors and accumulation in the brain and thymus of line cells. Eur. J. Immunol. 13:418-423.

3. Ben-nun, A., and I. R. Cohen. 1982. Spontaneous remission and acquired resistance to autoimmune encephalomyelitis (EAE) are associated with suppression of $\mathrm{T}$ cell reactivity: suppressed EAE effector $\mathrm{T}$ cells recovered as $\mathrm{T}$ cell lines. J. Immunol. 128:1450-1457.

4. Werdelin, O., and R. T. McCluskey. 1971. The nature and the specificity of mononuclear cells in experimental autoimmune inflammations and the mechanisms leading to their accumulation. J. Exp. Med. 133:1242-1263.

5. Stohl, W., and N. K. Gonatas. 1978. Chronic permeability of the central nervous system to mononuclear cells in experimental allergic encephalomyelitis in the Lewis rat. J. Immunol. 120:844-850.

6. Najarian, J. S., and J. D. Feldman. 1963. Specificity of passively transferred delayed type hypersensitivity. J. Exp. Med. 118:3421-3452.

7. Cohen, J. A., D. M. Issayan, B. Zweiman, and R. P. Lisak. 1987. Limiting dilution analysis of the frequency of antigen reactive lymphocytes isolated from the central nervous system of Lewis rats with experimental autoimmune encephalomyelitis. Cell. Immunol. 108:203-213.

8. Hickey, W. F., B. L. Hsu, and H. Kimura. 1991. T lymphocyte entry into the central nervous system. J. Neurosci. Res. 28:254-260.

9. van Eden, W., J. E. R. Thole, R. van der Zee, A. Noordzig, J. D. A. van Embden, E. J. Hensen, and I. R. Cohen. 1988. Cloning of the mycobacterial epitope recognized by $\mathrm{T}$ lymphocytes in adjuvant arthritis. Nature (Lond.). 331:171-173.

10. Elias, D., D. Markovits, T. Reshef, R. van der Zee, and I. R. Cohen. 1990. Induction and therapy of autoimmune diabetes in the non-obese diabetic (NOD/ LT) mouse by a $65-\mathrm{kDa}$ heat shock protein. Proc. Natl. Acad. Sci. USA. 87:15761580.

11. Elias, D., T. Reshef, O. S. Birk, R. van der Zee, M. D. Walker, and I. R. Cohen. 1991. Vaccination against autoimmune mouse diabetes with a T-cell epitope of the human 65-kDa heat shock protein. Proc. Natl. Acad. Sci. USA. 88:3088-3091.

12. Antoniou, A. V., D. Parker, J. L. Turk, B. G. Tan, and R. J. Scheper. 1986. Immunocytochemical identification and quantitation of mononuclear cells in the meninges during the development of chronic relapsing experimental allergic encephalomyelitis (CREAE) in the guinea pig. Cell. Immunol. 97:386-396.

13. Lefkovitz, I., and H. Waldman. 1984. Limiting dilution analysis of cells of the immune system. I. The clonal basis of the immune response. Immunol. Today. 5:265-268.

14. Schluesener, H. J., and H. Wekerle. 1985. Autoaggressive T lymphocyte lines recognizing the encephalitogenic region of myelin basic protein: in vitro selection from unprimed rat T lymphocyte populations. J. Immunol. 135:31283133.

15. Munk, M. E., B. Schoel, S. Modrow, R. W. Karr, R. A. Young, and S. H. E. Kaufmann. 1989. T lymphocytes from healthy individuals with specificity to self epitopes shared by the mycobacterial and human $65 \mathrm{kDa}$ heat shock protein. J. Immunol. 143:2844-2849.

16. Fey, K., I. Melchers, and K. Eichmann. 1983. Quantitative studies on T cell diversity. IV. Mathematical analysis of multiple limiting populations of effector and suppressor T cells. J. Exp. Med. 158:40-52.

17. MacDonald, H. R., J. C. Cerottini, J. E. Ryser, J. L. Maryaneski, C. Taswell, M. B. Widmer, and K. T. Brunner. 1980. Quantitation and cloning of cytolytic T lymphocytes and their precursors. Immunol. Rev. 51:93-123.

18. Sgroi, D., R. N. Cohen, E. G. Lingenheld, M. Kathryn Strong, T. Binder, I. Goldshneider, D. Greiner, M. Grunnet, and R. B. Clark. 1986. T cell lines derived from the spinal cords of mice with experimental allergic encephalomyelitis are self-reactive. J. Immunol. 137:1850-1854.

19. Matsumoto, Y., K. Kawai, Y. Tomita, and M. Fugiwara. 1990. Limiting dilution analysis of the frequency of myelin basic protein reactive $T$ cells in Lewis, PVG/c and BN rats: implication for the susceptibility to autoimmune encephalomyelitis. Immunology. 69:215-221.

20. Fallis, R. J., M. L. Powers, M. S. Sy, and H. L. Weiner. 1987. Adoptive transfer of murine chronic relapsing autoimmune encephalomyelitis, analysis of 
basic protein reactive cells in lymphoid organs and nervous system of donor and recipient animals. J. Neuroimmunol. 14:205-219.

21. Kaufmann, S. H. E., U. Vath, J. E. R. Thole, J. D. A. van Embden, and F. Emmrich. 1987. Enumeration of T cells reactive with Mycobacterium tuberculosis organisms and specific for the recombinant mycobacterial 64 kilodalton protein. Eur. J. Immunol. 17:351-357.

22. Modlin, R. L., J. Melancon-Kaplan, S. M. M. Young, C. Pirmez, H. Kino, J. Convit, T. H. Rea, and B. R. Bloom. 1991. Learning from lesions: patterns of tissue inflammation in leprosy. Proc. Natl. Acad. Sci. USA. 85:1213-1217.

23. Dayan, C. M., M. Londei, A. E. Corcoran, B. Grubeck-Loebenstein, R. F. L. James, B. Rapoport, and M. Feldmann. 1991. Autoantigen recognition by thyroid-infiltrating T cells in Graves disease. Proc. Natl. Acad. Sci. USA 88:7415-7419.

24. Res, P. C. M., C. G. Schaar, F. C. Breedveld, W. van Eden, J. D. A. van Embden, I. R. Cohen, and R. R. P. de Vries. 1988. Synovial fluid T cell reactivity against $65 \mathrm{KD}$ heat shock protein of mycobacteria in early chronic arthritis. Lancet. 2:478-480.

25. Koga, T., A. Wand-Wurttenberger, J. de Bruyn, M. E. Munk, B. Schoel, and S. H. E. Kaufmann. 1989. T cells against a bacterial heat shock protein recognize stressed macrophages. Science (Wash. DC) 246:1112-1115.
26. Polla, B. S. 1988. A role for heat shock proteins in inflammation? Immunol. Today. 9:134-137.

27. Cohen, I. R., and D. B. Young. 1991. Autoimmunity, microbial immunity and the immunological homonculus. Immunol. Today. 12:105-110.

28. Selmaj, K., C. F. Brosnan, and C. S. Raine. 1991. Colocalization of lymphocytes bearing $\gamma \delta \mathrm{T}$ cell receptors and heat shock protein hsp $65+$ oligodendrocytes in multiple sclerosis. Proc. Natl. Acad. Sci. USA. 88:6452-6456.

29. Haregewoin, A., G. Soman, R. C. Hom, and R. W. Finberg. 1989. Human gamma-delta $\mathrm{T}$ cells respond to mycobacterial heat-shock protein. Nature (Lond.) 340:309-312

30. Stamenkovic, I., M. Stegagno, K. A. Wright, S. M. Krane, E. P. Amento, R. B. Colvin, R. J. Duquesnoy, and J. T. Kurnick. 1988. Clonal dominance among T lymphocyte infiltrates in arthritis. Proc. Natl. Acad. Sci. USA. 85:11791183.

31. Haskins, K., M. Portas, B. Bergmann, K. Lafferty, and B. Bradley. 1989. Pancreatic islet specific T cell clones from nonobese diabetic mice. Proc. Natl. Acad. Sci. USA. 86:8000-8004.

32. Hafler, D. A., A. D. Duby, S. J. Lee, D. Benjamin, J. C. Seidman, and H. L. Weiner. 1988. Oligoclonal T lymphocytes in the cerebrospinal fluid of patients with multiple sclerosis. J. Exp. Med. 167:1313-1322. 